\title{
An Appliance Impact Estimation on Power Quality Parameters in Microgrid Environment ${ }^{+}$
}

\author{
Vojtěch Blažek 1,2, Michal Petružela 1,2, Tomáš Vantuch ${ }^{2,3}$, Stanislav Mišák ${ }^{1,2}$, Zdenek Slanina ${ }^{4}$ \\ and Wojciech Walendziuk ${ }^{5, *}$ \\ 1 Department of Electrical Power Engineering, VSB Technical University of Ostrava, 17.listopadu 15, \\ 70800 Ostrava, Czech Republic; vojtech.blazek@vsb.cz (V.B.); michal.petruzela@vsb.cz (M.P.); \\ stanislav.misak@vsb.cz (S.M.) \\ 2 CENTRE ENET at VŠB, VSB Technical University of Ostrava, 17.listopadu 15, 70800 Ostrava, \\ Czech Republic; tomas.vantuch@vsb.cz \\ 3 Department of Computer Science at Faculty of Electrical Engineering and Computer Science, \\ VSB Technical University of Ostrava, 17.listopadu 15, 70800 Ostrava, Czech Republic \\ 4 Department of Cybernetic and Biomedical Engineering, VSB Technical University of Ostrava, \\ 17.listopadu 15, 70800 Ostrava, Czech Republic; zdenek.slanina@vsb.cz \\ 5 Faculty of Electrical Engineering, Bialystok University of Technology, Wiejska 45D, \\ 15-582 Bialystok, Poland \\ * Correspondence: w.walendziuk@pb.edu.pl; Tel.: +48-85-7469397 \\ + Presented at the 9th Innovations-Sustainability-Modernity-Openness Conference (ISMO'20), Bialystok, \\ Poland, 20-21 May 2020.
}

Published: 4 August 2020

\begin{abstract}
Nowadays Off-Grid systems driven by intelligent data analysis, predictive models and real-time optimizations are frequently entitled as Smart Grids. They represent a future step in a sustainable energy supply for applications at various scales, from small household configurations to the systems operating on a scale of larger areas, especially parts of a town or village. One of the key characteristics of such systems is the lower short-circuit power caused by a lower and mostly stochastic nature of their power source, which is represented by a renewable energy source. This phenomenon directly implies the difficulties of sustainable power quality (PQ). Our paper presents an analysis on the lowest possible level, where the impact of household appliances is examined towards a specific kind of response due to PQ parameter variation. The analysis was performed on 120 combinations of appliances. Each combination consisted of three appliances running simultaneously in the microgrid. The measured data were subjected to a statistical analysis. This analysis finds there are several kinds of appliances we need to focus on, in order to express the PQ parameter change of a given parameter. The results show that the microwave, AC heating and drill appliances had a high impact on the total harmonic distortion of voltage (THDV) disturbance. The microwave, AC heating and mower had the highest impact on the total harmonic frequency (FREQ) and the power factor (PF). For the sake of completeness, specific features of the given appliances were examined as for their relevance to the PQ disturbance, which revealed the most relevant features to be taken into account during the development of a predictive model.
\end{abstract}

Keywords: power quality; off-grid system; t-SNE; clustering; renewable energy sources

\section{Introduction}

Autonomous energy systems (so called Off-Grid systems) are systems supplied from renewable sources (RESs) and supported by energy storage. Different circumstances need to be considered in 
such energy source applications, when compared to the standard distribution and transmission systems. The stochastic and unstable character of RES on the part of a source causes the presence of a low short-circuit power, which decreases the stability of power quality (PQ) parameters in the OffGrid system [1,2]. The frequency (FREQ), voltage disturbance, total harmonic distortion of voltage (THDV) and current (THDC), and flicker severity belong to the most important PQ parameters [3]. It is necessary to keep these parameters within the requested limits to ensure a reliable and safe operation of appliances.

These different circumstances raised an interest in many researchers to develop optimization tools able to detect and backward optimize the PQPs to meet the requested limits according to the internationally defined standards and norms. Relevant algorithms are mentioned in [4,5]. The dominant approaches are machine learning-based algorithms, such as Support Vector Machines (SVM) [6,7], Artificial Neural Networks (ANN) [8,9], Genetic Algorithms (GA) [10] and their combinations. On the other hand, there are studies suggesting the widely known Wavelet Transformations (WT) [11] or Fuzzy based detection [12] to classify the power quality (PQ) failure from the learned patterns. Most of the studies, as it is concluded in [4,5], concentrate on the on-line detection and moderation of the effects of PQ decrease, but they do not focus on the prediction or avoidance of such events in the first place. While most of the available papers deal with the prediction of the total consumption or production [13], this paper examines the separate executions of several appliance combinations in order to examine their impact.2. Experiment Description

The main goal was to find out which appliances (Table 1) have a negative impact on PQ in the Off-Grid system. To test this, each time, we combined 3 different appliances to create a total of 120 combinations. These were switched on and off at defined intervals. The response of the system, in the view of PQ parameters, was measured and stored for statistical evaluations.

Table 1. Feature values of the applied appliances further used for their relevancy estimation.

\begin{tabular}{|c|c|c|c|c|c|c|c|c|c|}
\hline \multirow{2}{*}{ Appliance } & \multicolumn{3}{|c|}{ Load (W) } & \multirow{2}{*}{$\begin{array}{c}\begin{array}{c}\text { Power } \\
\text { Factor (-) }\end{array} \\
\text { avg } \\
\end{array}$} & \multicolumn{3}{|c|}{ Characteristic } & \multicolumn{2}{|c|}{ Power Supply } \\
\hline & avg & $\min$ & $\max$ & & Inductive & Capacitive & Resistive & Continuous & Switch \\
\hline Mower & 537.6 & 532.1 & 549.27 & 0.52 & True & False & False & True & False \\
\hline Drill & 157.1 & 149.5 & 167 & 0.49 & True & False & False & True & False \\
\hline Kettle & 619.1 & 617 & 628.3 & 1 & False & False & True & True & True \\
\hline Fridge & 207.6 & 195.5 & 219.5 & 0.72 & True & False & False & True & False \\
\hline Switched mode & 410 & 409.7 & 420.2 & 0.78 & False & True & False & True & True \\
\hline AC heating & 880 & 852.5 & 910 & 0.91 & True & False & False & True & False \\
\hline Microwave & 203 & 76.8 & 1348.3 & 0.84 & True & False & False & False & True \\
\hline Boiler & 307 & 305.8 & 346.5 & 0.99 & False & False & True & True & False \\
\hline TV & 44 & 42.8 & 50.5 & 0.6 & False & True & False & False & True \\
\hline Lights $^{1}$ & 156 & 152.5 & 165.1 & 0.84 & False & True & False & False & True \\
\hline
\end{tabular}

They revealed the fact that the appliances were responsible for irregular PQ behaviour, and which of their features were the most relevant for this prediction. This study may be used to design deeper feature engineering procedures in the tasks of $\mathrm{PQ}$ parameters, forecasting in order to increase their accuracy.

The selected appliances were combined into triplets resulting in 120 combinations. There were three connected appliances at once because single running appliances in a household are rather uncommon. Appliances were turned on for an equal time (12 $\mathrm{min})$ in our Off-Grid platform to measure the system's PQ parameters response. To omit the stochasticity produced by RES but keep the low short-circuit power, the system was supplied by charged batteries. Each combination of appliances resulted in a different State of Charge (SoC). The batteries were re-charged using a hybrid inverter to reach the same defined $\mathrm{SoC}$ after each triplet was measured. Re-charge lasted for $18 \mathrm{~min}$.

Figure 1 shows the Off-Grid system where 2 photovoltaic panels produce $2 \mathrm{kWp}$ per each under normal conditions. For the reasons mentioned before, all the energy consumed by appliances was supplied from the batteries. 


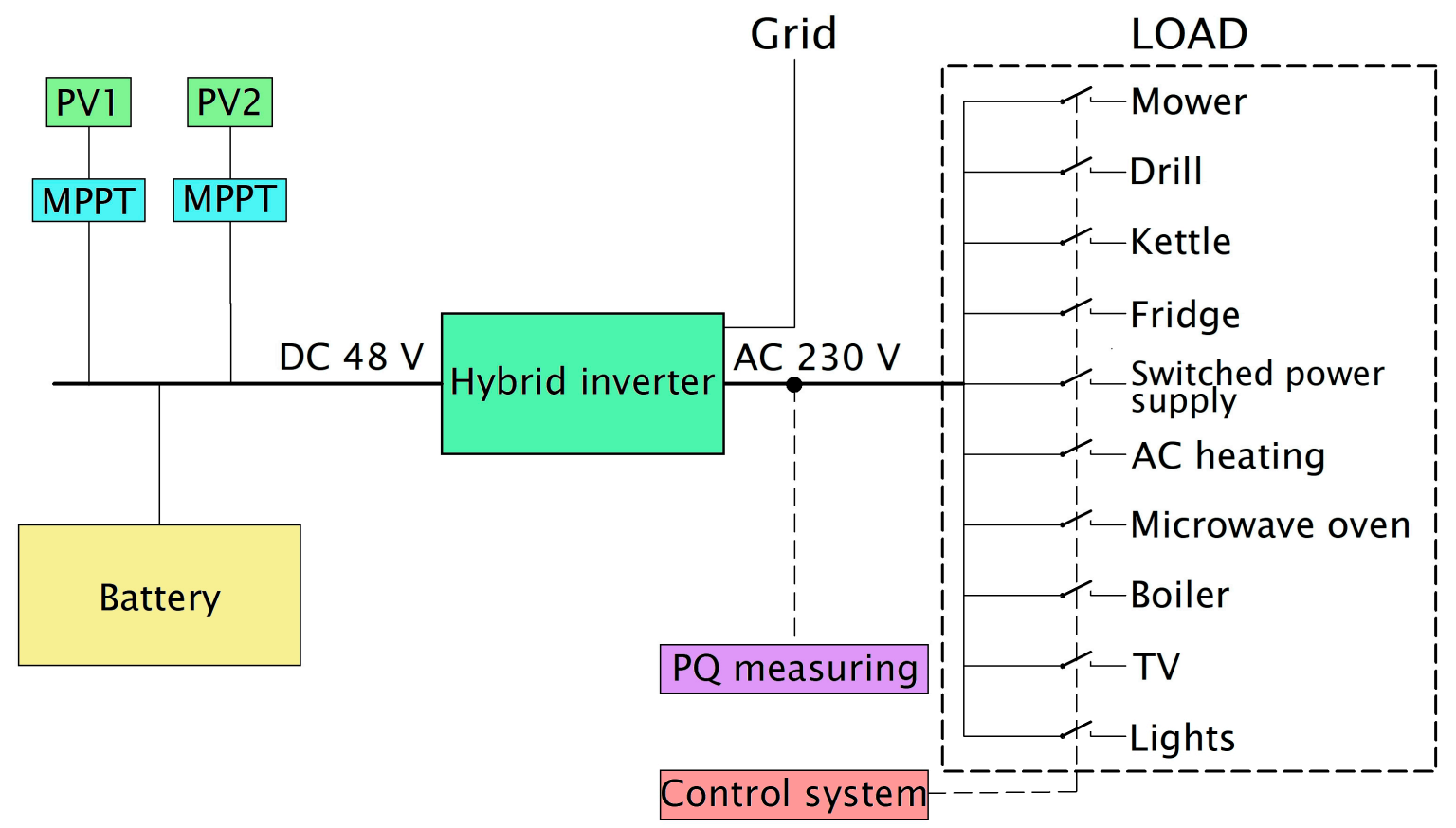

Figure 1. Scheme of an applied testing platform.

\section{Results and Conclusions}

The results of the experiments provided in this paper clearly distinguish the negative impact of certain appliances on the PQ parameters. The presence of the microwave significantly affected the behaviour of the frequency parameter. This resulted in a separated cluster that was confirmed and visualized by a Gap statistic-driven K-means algorithm.

A similar situation occurred in the case of the THDV parameter, where the appliances with a negative impact were the microwave, AC heating and drill. The last examined parameter was the power factor, the values of which were disturbed mostly by the mower, microwave and AC heating.

Very similar behaviour was observed in both cases, when batteries were fully and half charged. In the second case, the PQ disturbance was more significant, but the nature and source of the response remained the same.

Load value was estimated as the most relevant appliance feature for the PQP response. This has been directly evaluated by Kraskov MI estimation and later confirmed by the probabilities of the most significant appliances having the largest loads or the loads with the highest standard deviation.

Author Contributions: V.B. and M.P. Experiment design, V.B. Data providing and cleaning, M.P. and T.V. Writing-Original Draft Preparation, T.V. Statistical evaluation, V.B. and M.P. Hardware platform maintenance, S.M. Supervision and funding provider, Z.S. and W.W. Writing-Review \& Editing. All authors have read and agreed to the published version of the manuscript.

Acknowledgments: This paper was supported by the following projects: TN01000007 National Centre for Energy, TK02030039 Energy System for Grids, TH02020191 High Density Modular Power Controllers for Automotive and Aerospace Systems, SP2020/129 Research on Smart Grids Technologies and Components IIICENET. The research has been supported also by project WZ/WE-IA/2/2020 of the Bialystok University of Technology and financed from a subsidy provided by the Ministry of Science and Higher Education.

Conflicts of Interest: The authors declare no conflict of interest. 


\section{References}

1. Eckerbert, D.; Larsson-Edefors, P. Interconnect-driven short-circuit power modeling. In Proceedings of the IEEE Euromicro Symposium on Digital Systems Design, Warsaw, Poland, 4-6 September 2001; pp. 414421.

2. Saradarzadeh, M.; Farhangi, S.; Schanen, J.L.; Jeannin, P.O.; Frey, D. Combination of power flow controller and short-circuit limiter in distribution electrical network using a cascaded h-bridge distribution-static synchronous series compensator. IET Gener. Transm. Distrib. 2012, 6, 1121-1131.

3. Broshi, A. Monitoring power quality beyond en 50160 and iec 61000-4-30. In Proceedings of the IEEE 2007 9th International Conference on Electrical Power Quality and Utilisation, Barcelona, Spain, 9-11 October 2007; pp. 1-6.

4. Saini, M.K.; Kapoor, R. Classification of power quality events-A review. Int. J. Electr. Power Energy Syst. 2012, 43, 11-19.

5. Mahela, O.P.; Shaik, A.G.; Gupta, N. A critical review of detection and classification of power quality events. Renew. Sustain. Energy Rev. 2015, 41, 495-505.

6. Biswal, B.; Biswal, M.; Dash, P.K.; Mishra, S. Power quality event characterization using support vector machine and optimization using advanced immune algorithm. Neurocomputing 2013, 103, 75-86.

7. De Yong, D.; Bhowmik. S.; Magnago, F. An effective power quality classifier using wavelet transform and support vector machines. Expert Syst. Appl. 2015, 42, 6075-6081.

8. Valtierra-Rodriguez, M.; de Jesus Romero-Troncoso, R.; Osornio-Rios, R.A.; Garcia-Perez, A. Detection and classification of single and combined power quality disturbances using neural networks. IEEE Trans. Ind. Electron. 2014, 61, 2473-2482.

9. Raptis, T.; Vokas, G.; Langouranis, P.; Kaminaris, S. Total power quality index for electrical networks using neural networks. Energy Procedia 2015, 74, 1499-1507.

10. El-Naggar, K.M.; Al-Hasawi, W.M. A genetic based algorithm for measurement of power system disturbances. Electr. Power Syst. Res. 2006, 76, 808-814.

11. Nath, S.; Dey, A.; Chakrabarti, A. Detection of power quality disturbances using wavelet transform. World Acad. Sci. Eng. Technol. 2009, 49, 869-873.

12. Ibrahim, W.R.A.; Morcos, M.M. An adaptive fuzzy self-learning technique for prediction of abnormal operation of electrical systems. IEEE Trans. Power Deliv. 2006, 21, 1770-1777.

13. Vantuch, T.; Vidal, A.G.; Ramallo-González, A.P.; Skarmeta, A.F.; Misák, S. Machine learning based electric load forecasting for short and long-term period. In Proceedings of the 2018 IEEE 4th World Forum on Internet of Things (WF-IoT), Singapore, 5-8 February 2018; pp. 511-516.

(C) 2020 by the authors. Licensee MDPI, Basel, Switzerland. This article is an open access article distributed under the terms and conditions of the Creative Commons Attribution (CC BY) license (http://creativecommons.org/licenses/by/4.0/). 